06

\title{
Импульсное сжатие и растяжение эпоксидной смолы при ударно-волновом воздействии
}

\author{
(C) В.М. Мочалова, ${ }^{1,2}$ А.В. Уткин, ${ }^{1}$ А.В. Павленко, ${ }^{3}$ С.Н. Малюгина, ${ }^{3}$ C.С. Мокрушин ${ }^{3}$ \\ ${ }^{1}$ Институт проблем химической фризики РАН, \\ 142432 Черноголовка, Московская обл., Россия \\ ${ }^{2}$ Институт теоретической и экспериментальной фризики им. А.И. Алиханова Национального исследовательского центра \\ „Курчатовский институт“, \\ 117218 Москва, Россия \\ ${ }^{3}$ Российский федеральный ядерный центр - Всероссийский научно-исследовательский институт технической фризики \\ им. акад. Е.И. Забабахина, \\ 456770 Снежинск, Челябинская обл., Россия \\ e-mail: roxete20000@mail.ru
}

(Поступило в Редакцию 23 марта 2018 г.)

C использованием многоканальных лазерных интерферометров VISAR и PDV проведены исследования ударно-волновых свойств эпоксидной смолы ЭД-10. Получены ударная адиабата эпоксидной смолы,зависимость скорости звука от давления и показано,что структура фронта ударной волны соответствует модели вязко-упругого тела максвелловского типа с характерным временем релаксации касательных напряжений около $0.3 \mu \mathrm{s}$. Измерены значения откольной прочности и обнаружена их зависимость от скорости деформации образца при импульсном растяжении.

DOI: 10.21883/JTF.2019.01.46973.122-18

\section{Введение}

Эпоксидные смолы находят широкое практическое применение в качестве связующего компонента, входящего в состав композиционных материалов. Они в значительной степени определяют свойства этих материалов, в частности, их отклик на ударно-волновые воздействия. Опубликованные литературные данные свидетельствуют о достаточно сложном характере деформирования эпоксидной смолы в условиях импульсного нагружения [1-8]. Подобно полиметилакрилату при низких давлениях отчетливо выраженное вязко-упругое поведение максвелловского типа с величиной сдвигового напряжения, достгающего $0.7 \mathrm{GPa}[1-3]$. При давлении выше $18 \mathrm{GPa}$ наблюдаются увеличение ударной сжимаемости [4], что интерпретируется как фазовый переход первого порядка. В то же время авторами [5] становится под сомнение существование этого фазового перехода и отмечается необходимость получения более точной экспериментальной информации. Практически отсуттствуют экспериментальные данные об откольной прочности эпоксидной смолы, реализующейся при импульсном растяжении. Поэтому актуальной задачей является получение экспериментальной информации об ударноволновых свойствах эпоксидной смолы.

\section{1. Схема экспериментов}

В опытах исследовалась эпоксидная смола ЭД-10 с молекулярным весом 660-860. Плотность образцов составляла $1.21 \mathrm{~g} / \mathrm{cm}^{3}$, измеренная скорость звука $c_{l}=2.63 \mathrm{~km} / \mathrm{s}$. Образцы толщиной от 4.3 до $9.4 \mathrm{~mm}$ и диаметром от 40 до $90 \mathrm{~mm}$ были вырезаны из единого блока.

Схема экспериментов по исследованию ударноволновых свойств эпоксидной смолы приведена на рис. $1, a$. Разгон ударников 1 осуществлялся либо взрывными метательными устройствами, либо легкогазовой пушкой. Во взрывных экспериментах ударные волны в исследуемых образцах 3 формировались при соударении алюминиевого ударника 1 диаметром $90 \mathrm{~mm}$, разогнанного продуктами взрыва до скорости $W_{i}$, с экраном 2. Интерферометром VISAR измерялась скорость движения границы образца с водой 4. Зондирующее излучение отражалось от алюминиевой фольги толщиной $7 \mu \mathrm{m}$, наклеенной на образец 5. Для определения абсолютного значения скорости отраженное излучение регистрировалось двумя интерферометрами с постоянными 280 и $1280 \mathrm{~m} / \mathrm{s}$. В каждом опыте поляризационным датчиком 6 фиксировался момент входа ударной волны в образец, что с использованием интерферометрических данных позволяло определить значение волновой скорости $D_{s}$.

В опытах с использованием легкогазовой пушки ударник диаметром $34 \mathrm{~mm}$ соударялся непосредственно с образцом, экран 2 отсутствовал. Лазерными интерферометрами VISAR и PDV регистрировалась скорость свободной поверхности образца. При этом скорость в центре образца измерялась двухканальным интерферометром VISAR с различными постоянными скорости, а 8-канальный PDV использовался для определения пространственного распределения скорости на расстоянии 4 и $8 \mathrm{~mm}$ от центра.Схема расположения точек, в которых измерялась скорость, показана на рис. $1, b$. 

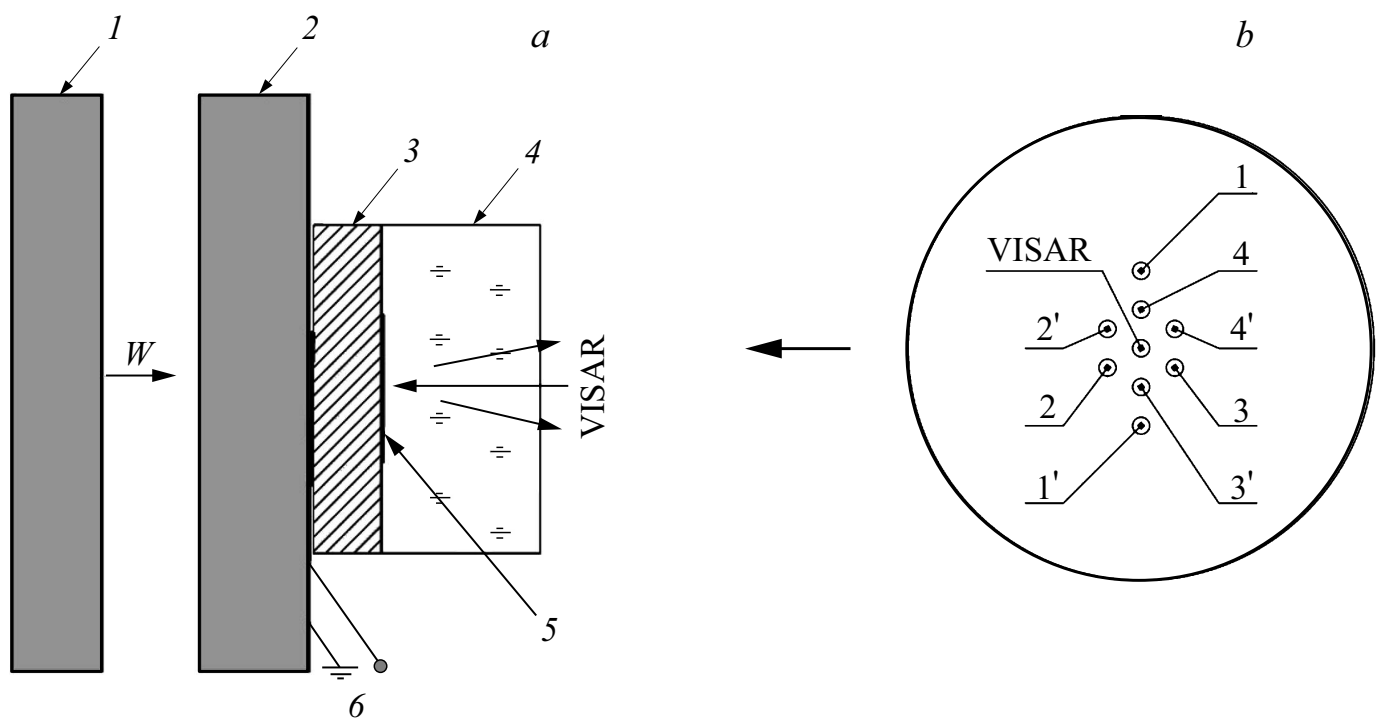

Рис. 1. $a-$ схема экспериментов по регистрации массовой скорости в эпоксиднойсмоле: $1-$ ударник, $2-$ экран, $3-$ исследуемый образец, 4 - граница образца с водой, 5 - алюминиевая фольга, 6 - поляризационный датчик; $b-$ схема расположения точек регистрации скорости свободной поверхности образцов в опытах с легкогазовой пушкой: в точках $1-4$ и $1^{\prime}-4^{\prime}$ измерения осуществлялись интерферометром PDV.

\section{1. Ударная сжимаемость}

Исследование ударной сжимаемости эпоксидной смолы осуществлялась в опытах со взрывными метательными устройствами.Параметры экспериментальных сборок и результаты экспериментов приведены в табл. 1 и на рис. 2,3 . В табл. 1 указаны скорость алюминиевого ударника $W_{i}$ и его толщина $h_{i}$, материал и толщина экрана $h_{b}$, измеренная скорость ударной волны $D$, давление $P$, массовая скорость $u$ и скорость звука $c$ в образце. Давление и массовая скорость в опытах с взрывными генераторами были рассчитаны по измеренным значениям $W_{i}, D$ и известным ударным адиабатам экрана. В опытах 5,6, проведенных на легкогазовой пушке, скорость ударной волны не измерялась. На рис. 2, 3 приведены полученные профили скорости, обозначения которых совпадают с нумерацией экспериментов в табл. 1 .

В опытах 1-4 (см. рис. 2) регистрируется резкий скачок скорости, за которым она остается практически постоянной до момента прихода волны разрежения со стороны ударника, отмеченного вертикальными стрелками. Время отсчитывается от момента входа ударной волны в образец. Профили скорости входящих волн измерялись в отдельных экспериментах на границе экрана с водой.Три из них, соответствующие опытам 1,2 и 4 и обозначенные как $1^{\prime}, 2^{\prime}$ и $4^{\prime}$, показаны на рис. 2 тонкими линиями. Заниженные значения амплитуд скорости на границеэкран вода по сравнению со скоростью границы образец/вода обусловлены различной ударно-волновой сжимаемостью воды и эпоксидной смолы.

В опытах 5, 6 амплитуда массовой скорости в несколько раз ниже, чем в опыте 1 (скорость на границе с водой в опытах 1-4 превышает массовую не более, чем

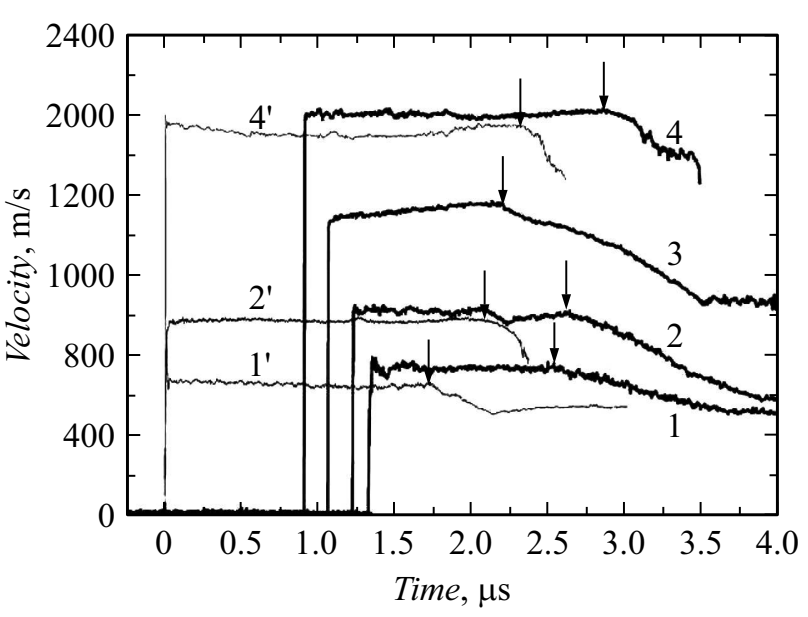

Рис. 2. Профили массовой скорости на границе с водой в опытах с применением взрывных метательных устройств.

на 20\%, тогда как скорость свободной поверхности в опытах 5,6 равна удвоенной массовой скорости). В результате более отчетливо проявляется структура фронта ударной волны. На рис. 3 видно, что после ударного скачка до $\sim 170 \mathrm{~m} / \mathrm{s}$ в опыте 5 и до $\sim 400 \mathrm{~m} / \mathrm{s}$ в опыте 6 скорость продолжает плавно возрастать в течение примерно $0.3 \mu$ s до своего максимального значения. Такой характер изменения параметров за ударной волной соответствует модели вязко-упругого тела максвелловского типа, которая базируется на описании релаксации касательных напряжений при деформировании среды.

На рис. 3 для каждого из опытов 5,6 представлены четыре профиля скорости, зарегистрированные в центре образца (VISAR) и на различном расстоянии от центра 
Таблица 1. Параметры и результаты экспериментов при определении ударной адиабаты эпоксидной смолы

\begin{tabular}{c|c|c|c|c|c|c|c|c}
\hline No & $\begin{array}{c}W_{i} \\
\mathrm{~km} / \mathrm{s}\end{array}$ & $\begin{array}{c}h_{i}, \\
\mathrm{~mm}\end{array}$ & $\begin{array}{c}h_{b}, \\
\mathrm{~mm}\end{array}$ & $\begin{array}{c}h_{s}, \\
\mathrm{~mm}\end{array}$ & $\begin{array}{c}\mathrm{D}, \\
\mathrm{km} / \mathrm{s}\end{array}$ & $\begin{array}{c}\mathrm{u}, \\
\mathrm{km} / \mathrm{s}\end{array}$ & $\begin{array}{c}\mathrm{P}, \\
\mathrm{GPa}\end{array}$ & $\begin{array}{c}c, \\
\mathrm{~km} / \mathrm{s}\end{array}$ \\
\hline 1 & 1.10 & 7 & $\mathrm{Cu}, 5.5$ & 4.40 & $3.31 \pm 0.05$ & $0.61 \pm 0.02$ & $2.44 \pm 0.05$ & $4.67 \pm 0.25$ \\
2 & 1.10 & 7 & $\mathrm{Al}, 4.0$ & 4.40 & $3.60 \pm 0.05$ & $0.88 \pm 0.02$ & $3.81 \pm 0.05$ & $5.44 \pm 0.30$ \\
3 & 2.50 & 10 & $\mathrm{Cu}, 8.0$ & 4.40 & $4.17 \pm 0.05$ & $1.38 \pm 0.02$ & $6.80 \pm 0.05$ & - \\
4 & 2.50 & 10 & $\mathrm{AL}, 4.0$ & 4.40 & $4.84 \pm 0.05$ & $1.86 \pm 0.02$ & $10.6 \pm 0.05$ & $6.77 \pm 0.30$ \\
5 & 0.167 & 3.92 & - & 4.58 & - & $0.13 \pm 0.005$ & - & - \\
6 & 0.376 & 3.94 & - & 4.62 & - & $0.31 \pm 0.005$ & - & -
\end{tabular}

(PDV).В скобках через запятую указаны точки,в которых осуществлялось измерение интерферометром PDV в опытах 5 и 6 соответственно. В пределах точности измерения профили совпадают. Это свидетельствует как о высокой степени плоскостности ударника, нагружающего образец, так и об однородности исследуемого материала.

В результате обработки экспериментальных данных 1-4 построена ударная адиабата эпоксидной смолы, которая приведена на рис. 4 в координатах $D-u$ круглыми черными точками. В исследованном диапазоне давлений экспериментальные данные аппроксимируются зависимостью $D=2.6+1.18^{*} u, \mathrm{~km} / \mathrm{s}$ (прямая линия на рис. 4). Причем первый коэффициент этой зависимости с хорошей точностью совпадает со скоростью звука, измеренной при атмосферном давлении. Там же приведены результаты, полученные авторами $[1,7]$ для эпоксидных смол, начальная плотность и продольная скорость звука которых изменяются в диапазоне $1.14-1.19 \mathrm{~g} / \mathrm{cm}^{3}$ и $2.38-2.65 \mathrm{~km} / \mathrm{s}$ соответственно. Несмотря на некоторое различие начальных свойств, согласие полученных данных при значениях массовой скорости меньше $1 \mathrm{~km} / \mathrm{s}$ следует считать удовлетворительным.

Полученные экспериментальные данные в том случае,когда наряду с профилем скорости границы образец вода зарегистрирован входящий в образец импульс сжа-

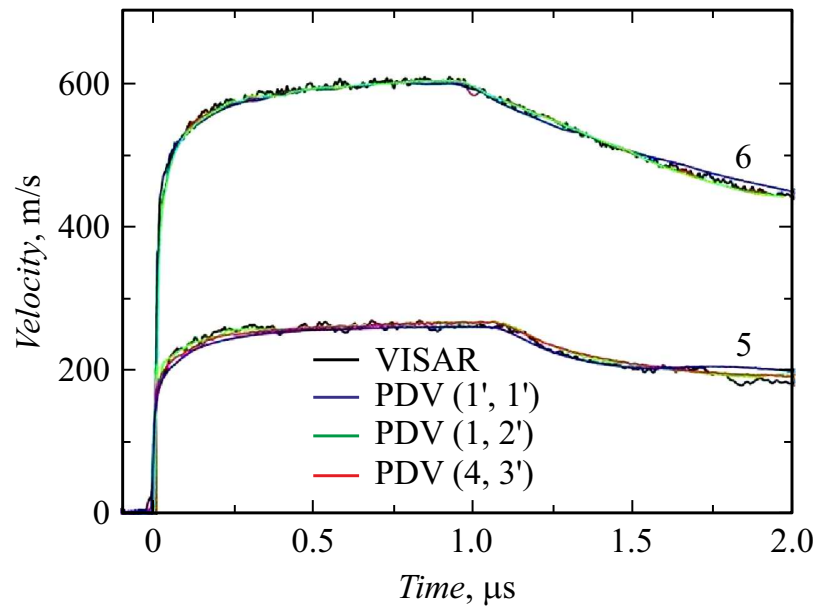

Рис. 3. Профили скорости свободной поверхности в опытах с легкогазовой пушкой.

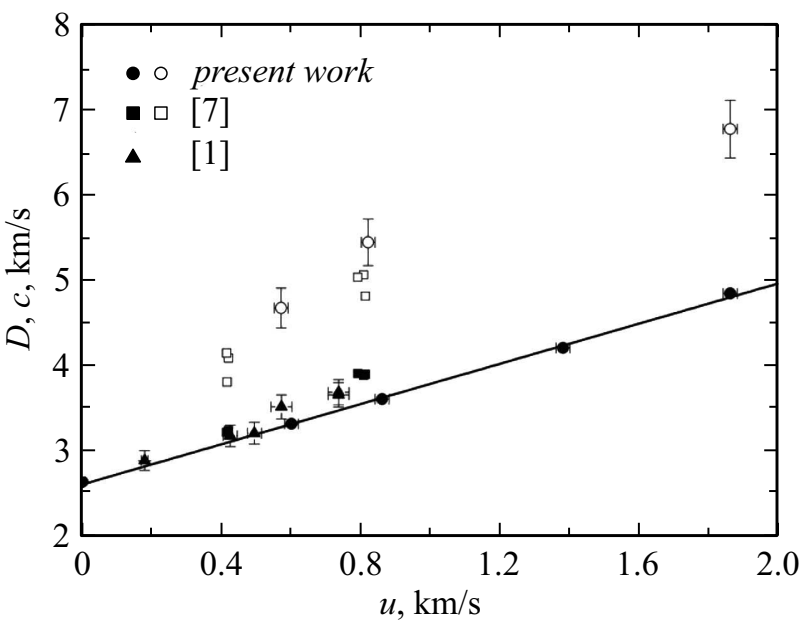

Рис. 4. Ударная адиабата эпоксидной смолы.

тия,позволяют определить зависимость скорости звука с от давления. Соответствующий анализ был выполнен методом характеристик [9] в предположении, что различие сжимаемостей воды и эпоксидной смолы не приводит к заметному искажению характеристик при взаимодействии падающей волны разгрузки с волной разгрузки, отраженной от границы эпоксидной смолы с водой. Точность определения скорости звука заметно хуже точности определения ударной адиабаты и, как правило, погрешность превышает 10\%. Результаты расчета c приведены на рис. 4 светлыми круглыми точками. Там же светлыми квадратами показаны значения скорости звука из работы [7], которые достаточно хорошо согласуются с полученными данными.

\section{2. Откольная прочность}

Определение прочности эпоксидной смолы при импульсном растяжении осуществлялось в условиях откольного разрушения [10]. Схема экспериментов аналогична приведенной на рис. $1, a$, но водяное окно отсутствовало, и разгрузка образца осуществлялась в воздух. В табл. 2 приведены параметры и результаты опытов с использованием взрывных метательных устройств (опыты 7-9), а также опыт 6, в котором также наблюдался откол. В опытах 7,8 алюминиевые 


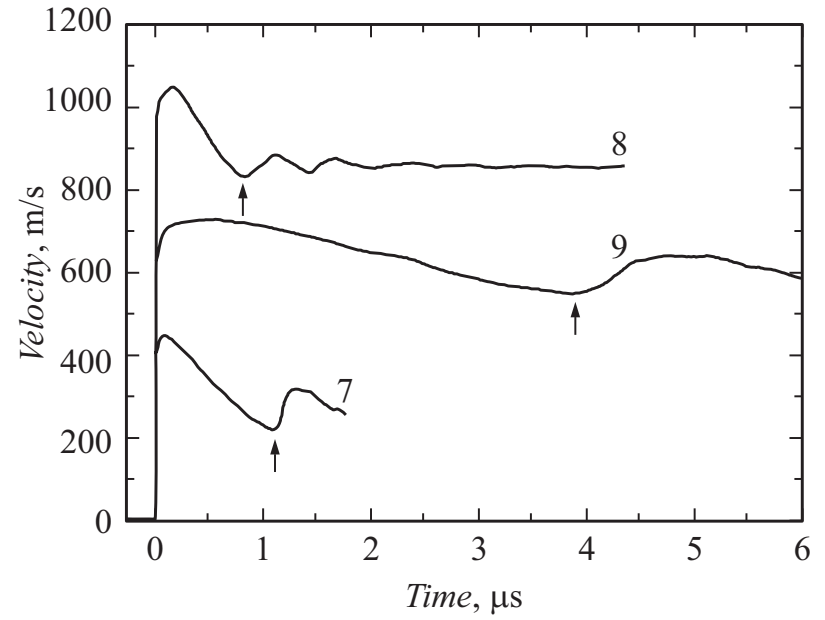

Pис. 5. Профили скорости свободной поверхности в опытах по определению откольной прочности эпоксидной смолы в экспериментах с использованием взрывных метательных устройств.

ударники соударялись непосредственно с образцом, а в опыте 9 ударная волна формировалась плосковолновым взрывным генератором [10], который отделялся от образца стальным экраном. Измеренные профили скорости свободной поверхности показаны на рис. 5, 6 .

Выход на свободную поверхность ударной волны вызывает скачкообразное увеличение скорости поверхности до максимального значения $W_{0}$. Внутрь образца распространяется центрированная волна разрежения, которая, взаимодействуя с падающей волной разгрузки, приводит к внутреннему разрыву - отколу. В процессе разрушения расятгивающие напряжения релаксируют к нулю, формируя вону сжатия, которая выходит на свободную поверхность в виде откольного импульса. Отмеченные особенности наблюдаются на профилях скорости, приведенных на рис. 5,6. Величина откольной прочности $\sigma_{s}$, характеризующая максимальные растягивающие напряжения в образце, определялась по разности между $W_{0}$ и минимумом скорости $W_{m}$, который достигается перед откольным импульсом и отмечен на рис. 5,6 вертикальными стрелками. В работе [11] показано, что для упруго-пластического тела

$$
\sigma_{s}=\rho_{0} c_{l}\left(W_{0}-W_{m}\right) /\left(c_{0}+c_{l}\right),
$$

где $c_{0}$ - объемная скорость звука. Это соотношение является точным, если импульс сжатия перед выходом на свободную поверхность треугольный, т. е. разгрузка начинается непосредственно за ударным скачком. Опыты 7-9 удовлетворяют данному условию и рассчитанные по формуле (1) значения откольной прочности приведены в табл. 2. Объемная скорость звука $c_{0}$ была рассчитана в предположении,что коэффициент Пуассона для эпоксидной смолы равен 0.33 [1].

В опыте 6 (рис. 6), проведенном на легкогазовой пушке, также наблюдается откольное разрушение, но в данном случае сущестует несколько факторов, которые необходимо учитывать при опредлеении $\sigma_{s}$. Во-первых, как видно на рис. 6, минимум $W_{m}$ на профилях скорости достигается в различные моменты времени иимеет различные значения в зависимости от расстояния точки регистрации от цетра образца. Максимальное отличие от профиля скорости, измеренного интерферометром VISAR, наблюдается в наиболее удаленных точках 1 и 1'. Обусловлено это влиянием боковой разгрузки, котоаря достигает центральной части обарзца примерно через $4 \mu \mathrm{s}$ после выхода ударной волны на свободную поверхность. Разрушение происходит раньше, поскольку откольный импульс регистрируется примерно через $2 \mu \mathrm{s}$. Поэтому профиль скорости, измеренный в центре, соответствует предположению об одномерности течения, используемому в методике расчета $\sigma_{s}$. Это условие для точек, лежащих на периферии, нарушается. Так, например, в тчоки 1 и 1', которые максимально удалены от центра, боковая разгрузка приходит примерно через $1.5 \mu \mathrm{s}$, т.е. раньше начала разрушения. Поэтому профили скорости, измеренные интерферометром PVD, некорректно использовать для расчета откольной прочности из-за нарушения одномерности течения в зоне разрушения.

Второе обстоятельство связано с тем, что импульс сжатия в опыте 6 не является треугольным,время между фронтом ударной волны и началом разгрузки составляет микросекунду, что практически совпадает с временем циркуляции волн в откольной пластине. В [12] показано, что для подобных профилей скорости в соотношение (1) необходимо вводить поправку $\Delta \sigma$ :

$$
\Delta \sigma=\left.\frac{1}{2} \frac{d \sigma}{d t}\right|_{C_{-}} \delta\left(\frac{1}{c_{0}}-\frac{1}{c_{l}}\right),
$$

где $\delta$ - толщина откольной пластины, $d \sigma /\left.d t\right|_{C_{-}}-$ удвоенный градиент напряжения в разгрузочной части импульса. Более подробно влияние градиентов напряжения в падающей волне разрежения и во фронте откольного импульса анализируется в работе [13].

На основании сказанного откольная прочность для опыта 6 была рассчитана по профилю скорости, изме-

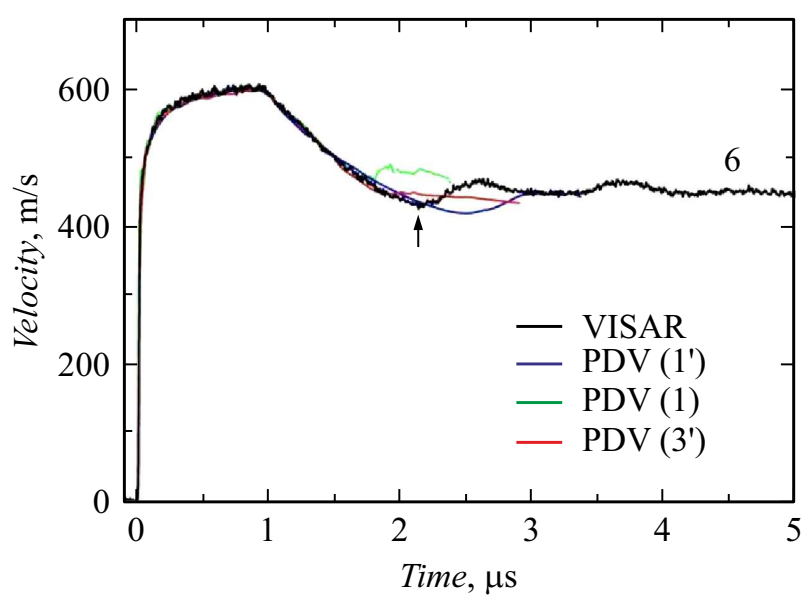

Рис. 6. Профили скорости свободной поверхности в опыте 6 при метании ударника легкогазовой пушкой. 
Таблица 2. Параметры и результаты экспериментов при определении откольной прочности эпоксидной смолы

\begin{tabular}{c|c|c|c|c|c|c}
\hline № & $\begin{array}{c}W_{i}, \\
\mathrm{~km} / \mathrm{s}\end{array}$ & $\begin{array}{c}h_{i}, \\
\mathrm{~mm}\end{array}$ & $\begin{array}{c}h_{b}, \\
\mathrm{~mm}\end{array}$ & $\begin{array}{c}h_{s}, \\
\mathrm{~mm}\end{array}$ & $\begin{array}{c}\rho_{s}, \\
\mathrm{MPa}\end{array}$ & $\begin{array}{c}\dot{\varepsilon} 10^{4}, \\
1 / \mathrm{s}\end{array}$ \\
\hline 6 & 0.376 & 3.94 & - & 4.62 & $285 \pm 5$ & $3.3 \pm 0.05$ \\
7 & 0.65 & 0.4 & - & 4.30 & $324 \pm 5$ & $5.0 \pm 0.10$ \\
8 & 0.65 & 2 & - & 4.75 & $314 \pm 5$ & $7.2 \pm 0.10$ \\
9 & - & - & $\begin{array}{c}\text { Сталь, } \\
20\end{array}$ & 9.40 & $257 \pm 5$ & $1.0 \pm 0.05$ \\
& & & &
\end{tabular}

ренному интерферометром VISAR в центральной части образца с учетом поправки (2), и приведена в табл. 2.

Отличительной особенностью полученных данных является влияние длительности исходного импульса сжатия на величину откольной прочности. Этот результат согласуется с общими представлениями о зависимости $\sigma_{s}$ от скорости деформации образца при растяжении [10]. В условиях откольного разрушения скорость деформации определяется по градиенту скорости свободной поверхности перед откольным импульсом [10]: $\dot{\varepsilon}=(d W / d t) / 2 c_{l}$. Значения $\dot{\varepsilon}$ приведены в табл. 2, и видно, что уменьшение скорости деформации в опыте 9 примерно в 5 раз по сравнению с опытами 7,8 приводит к снижению откольной прочности на 25\%.

\section{2. Обсуждение результатов}

Ударная сжимаемость исследованной эпоксидной смолы, как уже отмечалось,достаточно хорошо согласуется с данными авторов $[1,7]$ при низких давлениях. Однако при высоких давлениях наблюдается заметное расхождение $D-u$ зависимостей, особенно с данными [4]. Практически во всех работах ударная адиабата аппроксимируется линейным соотношением $D=a+b u$, причем коэффициент а, как правило, близок к продольной скорости звука. В то же время значение $b$ изменяется в широком диапазоне, от 1.115 [14] до 1.6 [4], что, очевидно, связано с различным составом эпоксидных смол. В эпоксидной смоле,как и в ПММА [15], за ударным скачком наблюдается релаксация ударносжатого вещества к равновесному состоянию.Поэтому можно говорить о равновесной и мгновенной ударной адиабате [15]. Поскольку при определении параметров ударного сжатия использовались значения массовой скорости,измеренные при временах,значительно превышающих характерное время релаксации, полученная ударная адиабата является равновесной.

Проведенные эксперименты показали, что эпоксидная смола ЭД-10 выдерживает достаточно высокие растягивающие напряжения. Величина откольной прочности в сопоставимых условиях ударно-волнового воздействия более чем на 15\% превышает прочность ПММА [16]. Наблюдается также заметный рост $\sigma_{s}$ при увеличении скорости деформации. Незначительное сниженное откольной прочности в опыте 8 , вероятно, обусловлено повышенной по сравнению с другими опытами остаточной температурой, поскольку амплитуда ударной волны и разогрев вещества в данном случае были максимальны.

Анализ профилей скорости свободной поверхности при отколе позволяет получить информацию, содержательную для разработки моделей разрушения при импульсном растяжении. Известно, например, что форма откольного импульса зависит от кинетики роста пор, возникающих на начальной стадии разрушения $[17,18]$. В этой связи представляет интерес сравнение профилей 7 и 8 на Рис. 5. Скорости деформации в разгрузочной части импульсов достаточно близки, тогда как градиент скорости во фронте откольного импульса в опыте 7 более чем в пять раз превышает соответствующую величину в опыте 8. Поскольку крутизна фронта откольного импульса определяется кинетикой роста пор [17], можно утверждать, что скорость роста пор в опыте 7 значительно выше. Основное различие этих опытов обусловлено амплитудными значениями ударных волн, а следовательно, остаточными температурами при которых происходит откольное разрушение. Если предположить, что скорость роста пор обусловлена вязкостью среды, то тогда вязкость должна возрастать с увеличением температуры, что маловероятно. Скорее всего, в данном случае следует говорить об изменении кинетики разрушения, например, о переходе от хрупкого механизма разрушения в опыте 7 к вязкому в опыте 8 .

\section{Заключение}

Таким образом, в результате проведенных экспериментов получена ударная адиабата эпоксидной смолы и показано,что структура фронта ударной волны соответствует модели вязко-упругого тела максвелловского типа с характерным временем релаксации касательных напряжений около $0.3 \mu \mathrm{s}$. Измерены значения откольной прочности и обнаружена их зависимость от скорости деформации образца при импульсном растяжении.Применение многоточечных интерферометров VISAR и PDV позволило получить пространственное распределение поля скоростей и исключить из анализа результаты,которые являются следствием нарушения одномерности течения волнами разгрузки с боковой поверхности образца.

Работа выполнена при поддержке Исследовательского центра ФАИР-Россия и по теме Государственного задания № 0089-2014-0017.

\section{Список литературы}

[1] Millett J.C.F., Bourne N.K., Barnes N.R. // J. Appl. Phys. 2002. Vol. 92. N 11. P. 6590-6594.

[2] Bourne N.K., Millett J.C.F., Barnes N., Belcher I. // Shock Compression of Condensed Matter. AIP Conference Proceedings. 2002. Vol. 620. N 1. P. 649-652. 
[3] Мержиевский Л.А., Воронин М.С. // Физика горения и взрыва. 2012. Т. 48. Вып. 2. С. 113-123. [Merzhievski L.A., Voronin M.S. // Combust. Explos. Shock Waves. 2012. Vol. 48. N 2. P. 226-235.]

[4] Carter W.J., Marsh S.P. Hugoniot Equation of State of Polymers, Report No. LA-13006-MS. 1995.

[5] Bordzilovskii S.A., Karakhanov S.M., Khishchenko K.V. // Combust. Explos. Shock Waves. 2013. Vol. 49. N 1. P. 121124.

[6] Bordzilovskii S.A., Karakhanov S.M., Merzhievskii L.A., Voronin M.S. // J. Appl. Phys. 2016. Vol. 120. P. 135903.

[7] Anderson M.U., Setchell R.E., Cox D.E. // Shock Compression of Condensed Matter. AIP Conference Proceedings. 2000. Vol. 505. N 1. P. 551-554.

[8] Krupnikov K.K., Krupnikova V.P. // Proceedings of the 19th International Symposium on Shock Waves in Condensed Matter and Heterogeneous Media. 1995. P. 301-306.

[9] Физика взрыва / Под ред Л.П. Орленко Т. 1. М.: Физматлит, 2002. $832 \mathrm{c}$.

[10] Канель Г.И., Разоренов С.В., Уткин А.В., Фортов В.Е. Ударно-волновые явления в конденсированных средах. М.: Янус-К, 1996. 248 c.

[11] Степанов Г.В. // Пробл. прочности. 1976. Вып. 8. С. 66-70.

[12] Романченко В.И., Степанов Г.В. // ПМТФ. 1980. Вып. 4. C. $141-147$.

[13] Канель Г.И. // ПМТФ. 2001. Т. 42. Вып. 2. С. 1-5.

[14] Трунин Р.Ф., Гударенко Л.Ф., Жерноклетов М.В., Симаков Г.В. Экспериментальные данные по ударно-волновому сжатию и адиабатическому расширению конденсированных веществ. Саров: РФЯЦ-ВНИИЭФ, 2001. 446 с.

[15] Barker L.M., Hollenbach R.E. // J. Appl. Phys. 1970. Vol. 41. N 10. P. 4208-4226.

[16] Канель Г.И., Разоренов С.В., Уткин А.В., Фортов В.Е. Экспериментальные профили ударных волн в конденсированных веществах. М.: Физматлит, 2008. 248 с.

[17] Уткин А.В. // ПМТФ. 1993. Т. 34. Вып. 4. С. 140-146.

[18] Уткин А.В. // ПМТФ. 2011. Т. 52. Вып. 1. С. 185-193. [Utkin A.V. // J. Appl. Mechan. and Techn. Phys. 2011. Vol. 52. N 1. P 151-158.] 